Ann. Biol. anim. Bioch. Biophys., I974, 14 (2), 371-375.

NOTE

\title{
DOSAGE DE L'HORMONE DE CROISSANCE (GH) PAR LE TEST TIBIA : IMPORTANGE DU CHOIX DES ANIMAUX
}

\author{
J. CHARRIER \\ avec la collaboration technique de Y. CARLES \\ Station de Physiologie animale, I. N. R. A., \\ Ecole nationale supérieure agronomique, \\ Place Viala, 34060 Montpellier Cedex
}

\section{RÉSUMÉ}

Cette étude porte sur l'influence de l'âge et du poids des rats utilisés dans la réalisation du " test tibia "pour le dosage biologique de l'hormone de croissance hypophysaire.

Il en découle que, pour une utilisation optimale du matériel animal et une bonne homogénéité de la réponse, ces rats doivent:

- être hypophysectomisés à l'âge de 32-34 jours ;

- avoir un poids de 83 à $93 \mathrm{~g}$;

- ne pas subir après hypophysectomie une variation de poids $\Delta \mathrm{P}$ dépassant les limites de $-3 \mathrm{~g}$ et de $+5 \mathrm{~g}$.

\section{INTRODUCTION}

Parmi les tests biologiques disponibles pour doser l'activité somatotrope, celui de l'augmentation en épaisseur du cartilage épiphysaire proximal du tibia de rat hypophysectomisé, dit " test tibia ", est un des plus couramment utilisés (Evans et al., I943; Greenspan et al., 1949; GeshWIND et LI, I955).

Les animaux employés par ces auteurs étaient hypophysectomisés à un âge allant de 26 à 42 jours bien que la plupart de leurs études aient été effectuées sur des rats opérés à 26-28 jours, stade généralement adopté depuis pour les dosages de routine.

Nous avons constaté que la réponse variait avec le poids et l'âge de l'animal.

La présente note résume cette étude. 


\section{MATÉRIEL ET MÉTHODES}

\section{A. - Animaux}

Les animaux sont des rats femelles IOPS $\left(^{1}\right)$ de la souche OFA $\left({ }^{2}\right)$ ayant tous le même âge pour un même lot et qui arrivent au laboratoire une semaine avant opération. Ils cohabitent par groupes de 8 dans des cages transparentes.

Tous les rats ont un accès libre et permanent à la nourriture. Ils logent dans une pièce où la température est de $30^{\circ} \pm \mathrm{I}^{\circ} \mathrm{C}$, température de neutralité thermique du rat (GIAJA et Gelrneo, I936). L'éclairement est donné de 7 à 2I heures par des tubes fluorescents.

\section{B. - Hypophysectomie}

L'hypophysectomie est pratiquée selon la technique de FaLconi et Rossi (I964), par voie transauriculaire, sous anesthésie à l'éther. Le contrôle de l'efficacité de l'opération est fait in vivo à partir des pesées corporelles et post-mortem par différents examens : absence de reliquats hypophysaires dans la selle turcique, involution des surrénales et du tractus génital.

Seuls ont été retenus les animaux pour lesquels l'efficacité de l'ablation a été vérifiée par l'ensemble de ces critères.

\section{C. - Autres caractéristiques techniques}

Les techniques de coloration des demi-tibias et de mesure des cartilages au microscope sont sensiblement les mêmes que celles décrites par GREENSPAN et al. (1949). L'exploitation statistique des résultats a été faite par analyses de variances selon FinNEY (I964).

\section{RÉSULTATS ET DISCUSSION}

\section{A. - Infuence de l'âge de l'opération}

La figure I montre l'évolution du poids des rats après hypophysectomie, selon qu'ils sont opérés à 28 jours (groupe A) ou à 34 jours (groupe B). Il s'avère que l'arrêt de croissance est immédiat chez les plus âgés, alors que la prise de poids, bien que ralentie, se poursuit encore quelques jours chez les plus jeunes.

\section{B. - Influence de la variation de poids $\Delta P$}

Pour savoir dans quelle mesure la variation de poids $\Delta \mathrm{P}$ après hypophysectomie intervient sur la réponse du cartilage épiphysaire à la $\mathrm{GH}$, l'ensemble des animaux des groupes A et B (fig. I) a reçu dans les conditions habituelles de l'expérience une dose totale de $320 \mu \mathrm{g}$ de BGH (Byla lot or/69).

Il apparaît que dans les deux groupes la $\Delta \mathrm{P}$ a une action sur la réponse du cartilage.

Dans les deux cas l'évolution de la réponse en fonction de $\Delta \mathrm{P}$ peut s'ajuster à une droite, les pentes de ces droites étant $b_{\mathrm{A}}=7,5$ et $b_{\mathrm{B}}=4,4$ donc nettement différentes (fig. 2). Dans le groupe $\mathrm{B}$ il est possible de déterminer une certaine zone de variation de $\Delta \mathrm{P}$, de $-3 \mathrm{~g}$ à $+5 \mathrm{~g}$, dont la pente est extrêmement faible $\left(b_{\mathrm{B}}^{\prime}=0,5\right)$, où l'on peut considérer que la réponse moyenne ne

(1) Indemnes d'Organismes Pathogènes Spécifiques. bresle.

(2) Centre de Recherche et d'Élevage Iffa-Credo, Domaine des Oncins, 69 Saint-Germain-sur-l'Ar- 


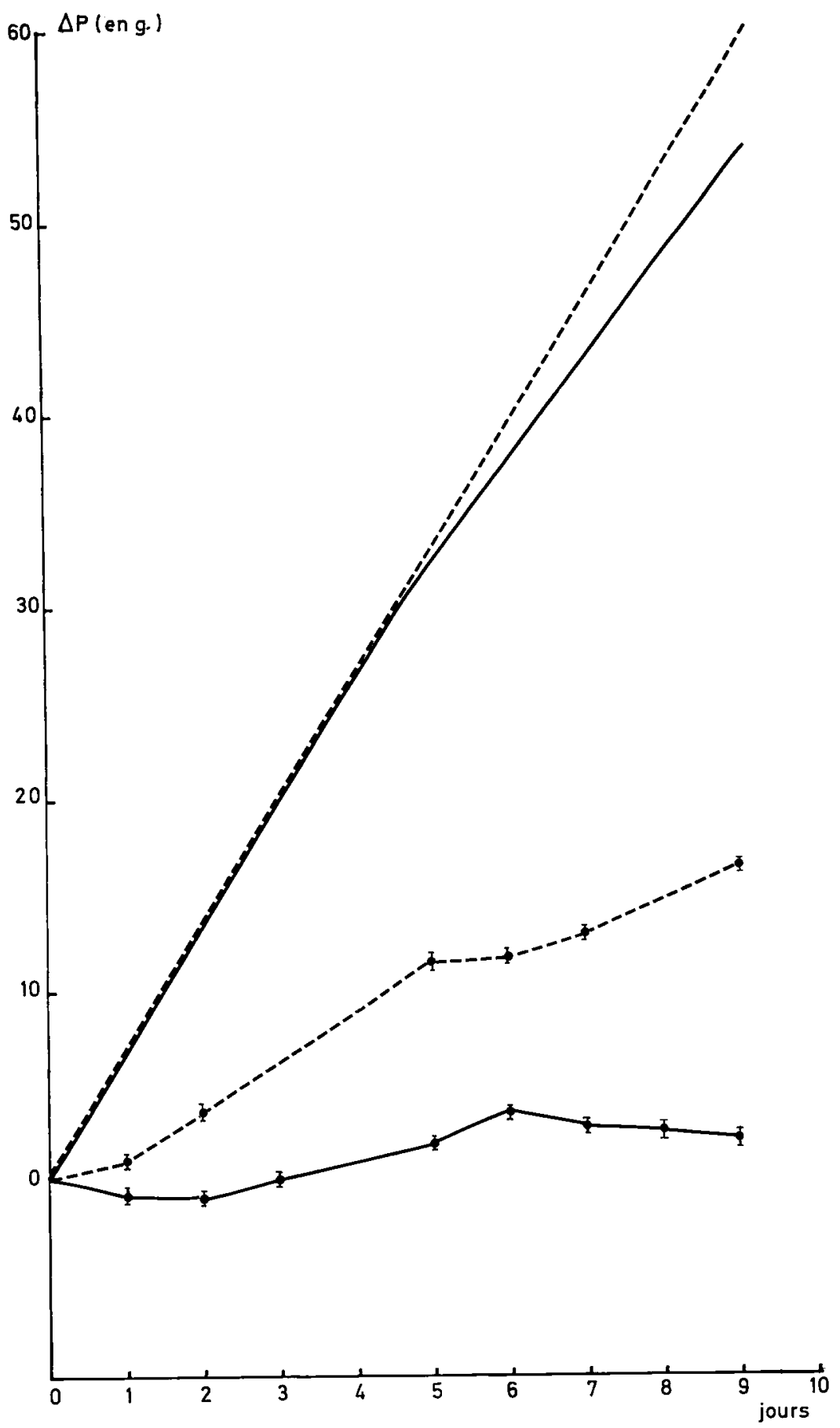

FIG. I. - Gain de poids quotidien $\Delta \mathrm{P}$ des rats après hypophysectomie

à 28 jours groupe A. Nombre d'animaux : I I7 - à 34 jours groupe $B$. Nombre d'animaux : I I 8

Pour chaque point : moyenne \pm erreur-type

En comparaison, évolution normale du poids de rats non opérés, à partir des mêmes âges : 
varie pas. L'existence d'une telle zone pour le groupe A n'apparait pas. Les erreurs types sont également nettement plus élevées pour $\mathrm{A}$ que pour $\mathrm{B}$.

De plus, en ne considérant que le groupe B, le poids initial avant opération permet d'effectuer une certaine discrimination par rapport à la variation de poids après hypophysectomie. Les limites pondérales de ce groupe allaient de 83 à I $55 \mathrm{~g}$. Parmi les animaux dont le poids à l'opération était $\leqslant 93 \mathrm{~g}, 84 \mathrm{p}$. Ioo entraient dans la fourchette retenue $(-3 \mathrm{~g}+5 \mathrm{~g})$, alors que parmi ceux dont le poids était $\geqslant 94 \mathrm{~g}, 56 \mathrm{p}$. roo seulement auraient été retenus.

En utilisant des animaux âgés de 34 jours et pesant de 83 à $93 \mathrm{~g}$ lors de l'hypophysectomie, on obtient une réponse à peu près constante (au moins pour la dose considérée).

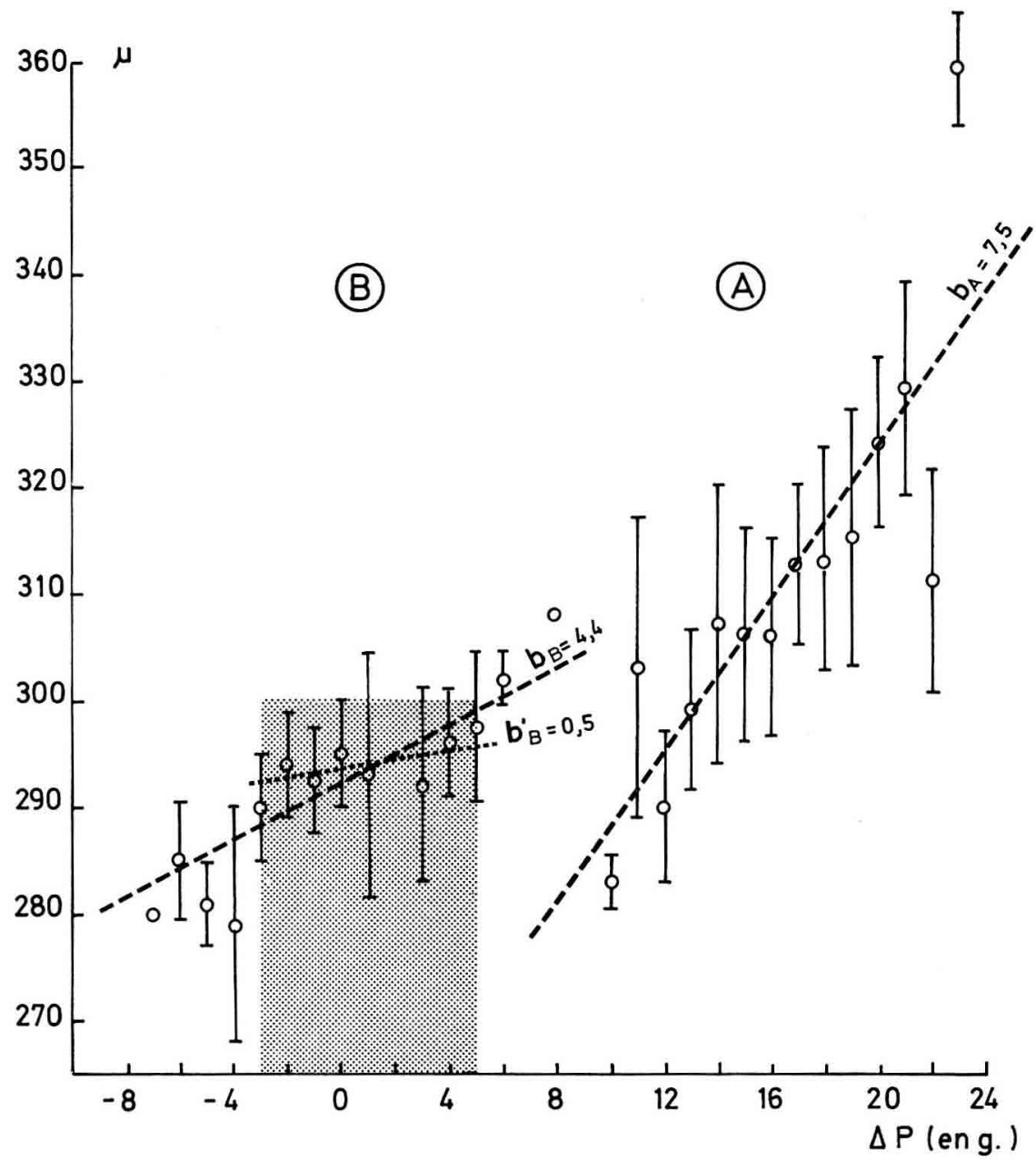

Fig. 2. - Réponse du cartilage (en $\mu$ ) à une dose totale de $320 \mu \mathrm{g}$ de $B G H$, en fonction de $\Delta \mathrm{P}$ avant injection 12 jours après hypophysectomie.

Groupes A et B comme figure I

Cette influence de l'âge à l'hypophysectomie peut être rapprochée des données de WALKER et al. (1950) disant que l'ablation de l'hypophyse n'arrêtait pas la croissance chez le Rat avant l'âge de 4 semaines environ. Déjà en 1942 MARX et al. avaient remarqué que la croissance pouvait 
continuer après l'hypophysectomie pendant quelques jours encore. C'est également ce que nous observons avec des animaux opérés à 28 jours, alors qu'après opération à 34 jours l'arrêt est immédiat. A notre avis le stade de $26-28$ jours représente probablement un âge critique moyen pouvant varier entre souches, mais certainement aussi à l'intérieur d'une même souche.

Pour un certain nombre d'individus ce seuil de sensibilité à l'hypophysectomie doit être repoussé de quelques jours. Ceci est important pour la sélection des animaux de dosage, car un rat opéré trop jeune physiologiquement aura une prise de poids qui pourrait affecter le dosage. Il est donc indispensable d'utiliser des animaux suffisamment âgés pour éviter ce genre d'inconvénient.

Reçu pour publication en octobre 1973.

\section{REMERCIEMENTS}

Ce travail a été réalisé en partie grâce au contrat D. G. R.S. T. nº 71-7-3 I 29 " Biologie de la Reproduction et du Développement postnatal".

\section{SUMMARY}

\section{DE'TERMINATION OF THE GROW'TH HORMONE (GH) BY MEANS} OF THE " TIBIA TEST ": IMPOR'TANCE OF THE CHOICE OF THE ANIMALS

This study concerns the influence of the age and weight of the rats submitted to the "tibia test "for the biological determination of the hypophyseal growth hormone.

The results lead to the conclusion that for an optimal utilization of the material and a good homogeneity in the response, the rats must :

- undergo an hypophysectomy when 32 to 34 days old;

- weigh 83 to $93 \mathrm{~g}$;

- undergo no weight variation $(\Delta \mathrm{P})$ exceeding $-3 \mathrm{~g}$ and $+5 \mathrm{~g}$ after hypophysectomy.

\section{RÉFÉRENCES BIBLIOGRAPHIQUES}

Evans H. M., Simpson M. E., Marx W., Kibrick E. A., I943. Bioassay of pituitary growth hormone. Width of the proximal epiphyseal cartilage of the tibia in hypophysectomized rats. Endocrinology, 32, 13-I6.

FAlconi G., Rossi G. L., I964. Transauricular hypophysectomy in rats and mice. Endocrinology, 74, 30r-303.

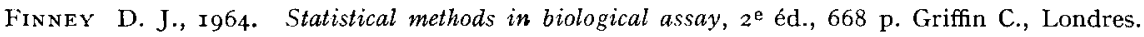

Geshwind I. I., Li C. H., I955. The tibia test for growth hormone in : Smith R. W., Gaebler O. H., and Long C. N. H., The hypophyseal growth hormone, nature and actions, 28-58, Mc Graw Hill, New York. Giaja J., Gelineo S., r936. Sur la courbe réelle de la thermogénèse des homéothermes. C. R. Soc. Biol., 122, 343-344.

Greenspan F. S., Li C. H., Simpson M. E., Evans H. M., I949. Bioassay of hypophyseal growth hormone : the tibia test. Endocrinology, 45, 455-463.

Marx W., Simpson M. E., Evans H. M., I944. Specificity of the epiphyseal cartilage test for the pituitary growth hormone. Proc. Soc. exp. Biol. Med., 55, 250-252.

Walker D. G., Srmpson M. E., Asling C. N., Evans H. M., I950. Growth and differenciation in the rat following hypophysectomy at six days of age. Anat. Rec., 106, 539-554. 